\title{
Hepatic Epithelioid Hemangioendothelioma with Cecal Metastasis in a Natural Course: A Case Report
}

\section{Ying-Ren Chen ${ }^{1}$, Chien-Hsien Lai ${ }^{2}$, Liang-Yi Hung ${ }^{2}$, Kung-Chao Chang ${ }^{1^{*}}$}

${ }^{1}$ Department of Pathology, National Cheng Kung University Hospital, College of Medicine, National Cheng Kung University, Tainan, Taiwan

2Institute of Bioinformatics and Bio signal Transduction, College of Bioscience and Biotechnology, National Cheng Kung University, Tainan, Taiwan

"Corresponding author: Kung-Chao Chang, Department of Pathology, College of Medicine, National Cheng Kung University and Hospital, 138 Sheng-Li Road, Tainan, 704, Taiwan. Tel: 886-6-235-3535 ext. 2636; Fax: 886-6-276-6195; E-mail: changkc@mail.ncku.edu.tw

Received date: Jan 29, 2016; Accepted date: Mar 08, 2016; Published date: Mar 18, 2016

Copyright: (c) 2016 Chen Y, et al. This is an open-access article distributed under the terms of the Creative Commons Attribution License, which permits unrestricted use, distribution, and reproduction in any medium, provided the original author and source are credited.

\begin{abstract}
Epithelioid hemangioendothelioma (EHE) is a rare vascular tumor of low malignant potential that occurs mostly in soft tissue. So far, only two cases of EHE involving the intestine have been recorded. Here, we describe a rare case of cecal EHE due to a subsequent metastasis from the primary liver tumor three years after initial diagnosis. A 74year-old man had sudden onset of epigastralgia for 24 hours. The abdominal CT revealed an ileocecal mass with a small bowel obstruction, and extensive tumor involvement in the liver was noted. He received an emergent right hemicolectomy for relief of the ileus. Unfortunately, he expired four days later due to septic shock. The pathologic diagnosis of EHE prompted a molecular study for a WWTR1-CAMTA1 fusion. The Sanger sequencing results showed the fusion involved exon 4 of WWTR1 with exon 8 of CAMTA1. There is no standard treatment for hepatic EHE because of its rarity and variable clinical outcome. The decision on a treatment strategy should be individualized for each patient. Since the patient received supportive care only for the liver tumor, this case demonstrated a natural course of hepatic EHE with a survival of more than 3.3 years.
\end{abstract}

Keywords: Cecal tumor; Epithelioid hemangioendothelioma; Liver; Metastasis; WWTR1-CAMTA1 gene fusion

\section{Introduction}

Epithelioid hemangioendothelioma (EHE) is an uncommon vascular tumor of low malignant potential and occurs mostly in soft tissue. In the literature, only two cases of EHE involving the intestine have been recorded $[1,2]$. Here, we report a rare case of cecal EHE, which represents a subsequent metastasis from the primary liver tumor, three years after the liver tumor diagnosis. Notably, since the patient received supportive care only for the liver tumor, this case demonstrated a natural course of hepatic EHE with a survival of more than 3.3 years.

\section{Case Report}

A 74-year-old man had a liver tumor diagnosed at a different hospital facility not related to NCKUH in April 2010. The patient presented with multiple hepatic masses and metastatic nodules in the lung at that time of initial diagnosis. The patient and his family refused aggressive treatment and chose to receive supportive care only. The follow-up images showed progression of hepatic and pulmonary tumors with metastasis to mediastinal and paracaval lymph nodes. On August 12, 2013 the patient presented to our emergency medicine department complaining of sudden onset epigastralgia from the previous 24 hours. An elevated alkaline phosphatase level (187 U/L, normal range, 30-100) with normal aminotransaminase liver enzyme profile was detected. Abdominal Computed Tomography (CT) revealed an ileocecal mass, measuring $4.0 \times 3.3 \mathrm{~cm}$ with a small bowel obstruction (Figure 1A arrow).

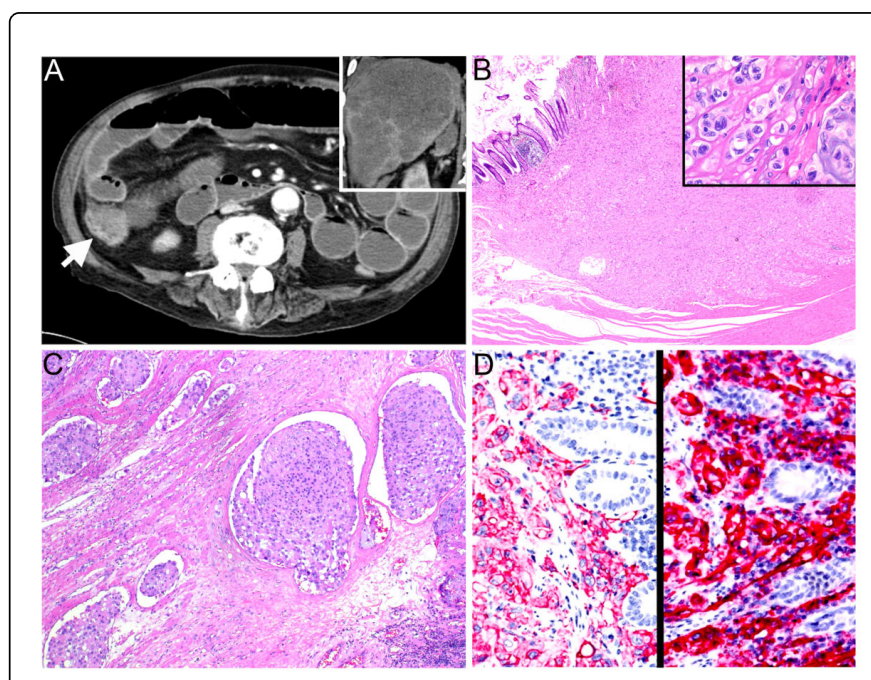

Figure 1: Clinicopathologic features of hepatic epithelioid hemangioendothelioma with cecal metastasis.(A) Abdominal CT reveals an ileocecal mass (arrow), measuring $4.0 \times 3.3 \mathrm{~cm}$ with small bowel obstruction. Extensive tumor involvement in the liver is noted (inset). (B) Microscopically, the tumor infiltrates the colonic wall with a focal mucosal involvement (H\&E, 20X), and is composed of cords or nests of epithelioid cells with characteristic intracytoplasmic vacuoles in a myxohyaline stroma (inset, $\mathrm{H} \& \mathrm{E}$, 400X). (C) Extensive tumor emboli are found in the sample (H\&E, 100X). (D) Immunohistochemically, the tumor cells are positive for CD34 (left panel) and CD31 (right panel). 
In addition, extensive tumor involvement in the liver was also noted (Figure 1A inset). For relief of the ileus, he received an emergent right hemicolectomy. Unfortunately, the post-operative course was complicated by septic shock with adult respiratory distress syndrome. The patient expired four days later.

Grossly, the specimen was a segment of the right side colon $(37 \mathrm{~cm}$ in length) containing a cecal mass $(3 \times 3 \times 2 \mathrm{~cm})$. The tissue sample was fixed in $10 \%$ neutral buffered formalin for 24 hours at room temperature and paraffin-embedded using standard procedures. Tissue sections were cut at $4 \mu \mathrm{m}$ and stained with hematoxylin and eosin (H\&E). Two independent pathologists interpreted the slides and described their findings. Microscopically, the tumor mainly infiltrated into the colonic wall and peri-colonic tissue with focal mucosal involvement (Figure 1B). It was composed of cords and nests of epithelioid tumor cells with characteristic intracytoplasmic vacuoles in a myxohyaline stroma (Figure 1B inset).

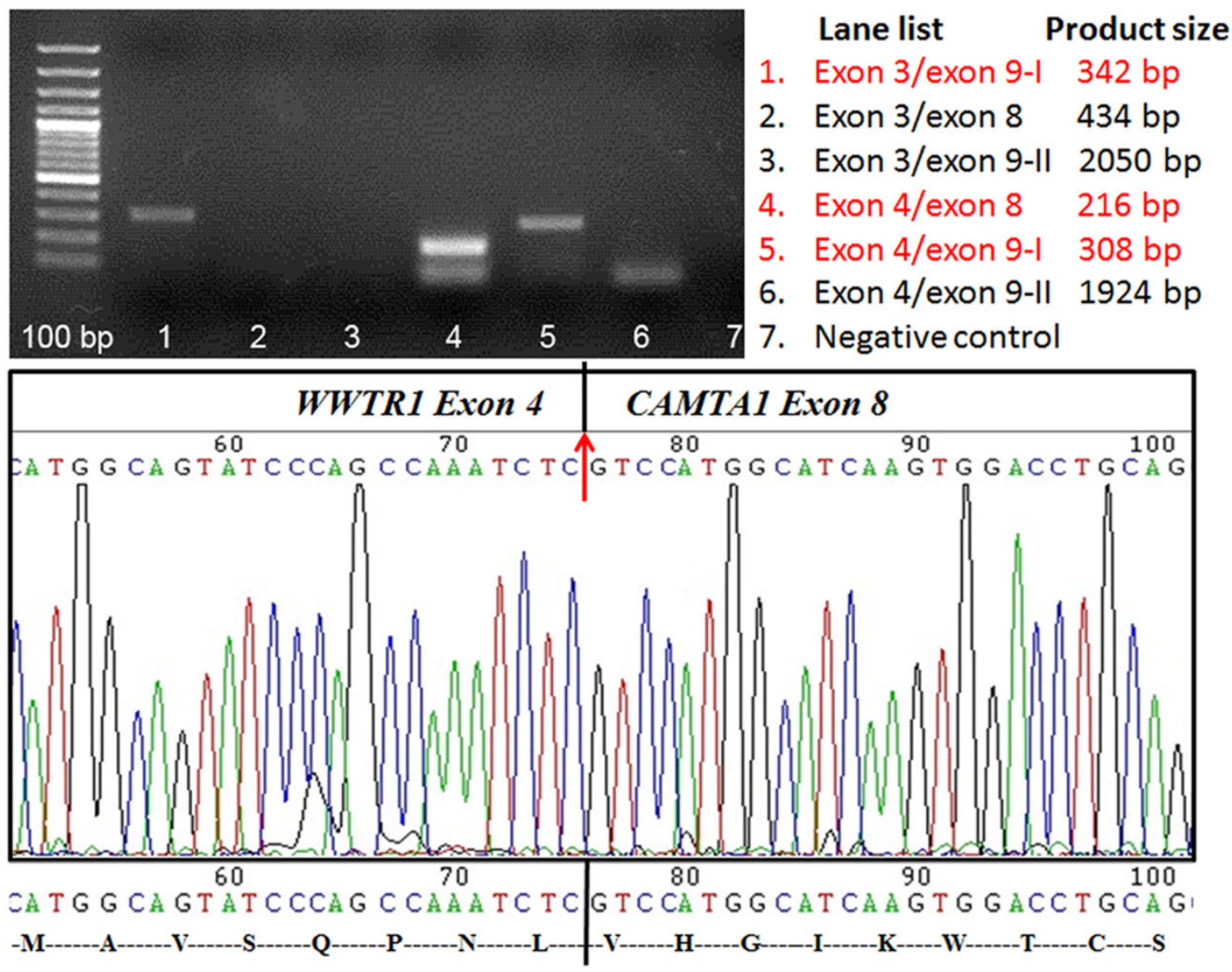

Figure 2: Molecular studies of the WWTR1-CAMTA1 gene fusion. Paraffin-embedded tumor tissues were used for RT-PCR to test for the WWTR1-CAMTA1 fusion transcript with six pairs of primers as listed. The positive results are labeled in red (upper right panel). Because the RNA molecules were fragmented in paraffin tissue, expected products more than $400 \mathrm{bp}$ were unamenable for detection (upper left panel lanes 2, 3 and 6). The lower bands in lanes 4 and 6 indicate non-specific PCR products. The fusion involves exon 4 of WWTR1 with exon 8 of CAMTA1 as detected by Sanger sequencing (lower panel).

Mitotic figures and necrotic foci were occasionally found. Extensive tumor emboli were discerned (Figure 1C), consistent with a metastatic nature. Since the tumor cells were epithelioid, the differential diagnosis may include carcinoma and epithelioid mesenchymal sarcoma, especially vascular tumors. The bland nuclear features, a low mitotic activity, and a slow progressive course would collectively argue against aggressive carcinoma or angiosarcoma. Utilizing a tissue autostainer (Bond-Max autostainer; Leica Biosystems Newcastle Ltd, Australia), immunohistochemical staining was performed on $4-\mu \mathrm{m}$-thick sections after tissues were deparaffinized with xylene and pre-treated with either Epitope Retrieval Solution 2 (EDTA, pH 9.0) or $10 \mathrm{mM}$ sodium citrate buffer ( $\mathrm{pH}$ 6.0). Hematoxylin was used as a counterstain. The primary antibodies were as follows: CD31 (JC70A, 1:100, Dako,
Glostrup, Denmark), CD34 (QBEnd-10, 1:50, Dako), pan cytokeratin (AE1/AE3, 1:200, Dako), and D2- 40 (D2-40, 1:25, MyBiosource, San Diego, CA, USA). Appropriate positive and negative controls were used. Immunohistochemically, the tumor cells were positive for CD34 (Figure 1D left panel), CD31 (Figure 1D right panel) and D2-40, but negative for pan cytokeratin stain. Under the impression of EHE, a molecular study by reverse transcriptase polymerase chain reaction (RT-PCR) confirmed the WWTR1-CAMTA1 gene fusion in paraffinembedded tumor tissue (Figure 2). After reviewing the liver tumor pathology, it showed similar morphology as the metastasis. Taken together with the clinical history, the diagnosis of hepatic EHE with cecal metastasis was made, an extremely rare case. 
Page 3 of 3

\section{Discussion}

Hepatic EHE is a rare vascular tumor, which originates from endothelial cells with an incidence of $<0.1$ per 100,000 population. It appears more often in women with a male-to-female ratio of $2: 3$ and a peak incidence in the 3rd to 4th decade of life [3]. Histologically, EHE appears as nests or cords of epithelioid endothelial cells with characteristic intracellular vascular lumina which sometimes contains red blood cells. Immunoreactivity for CD31, CD34, D2-40 and Factor VIII-related antigen provides further evidence of endothelial differentiation. EHE carries a disease-defining genetic mutation of $t$ $(1 ; 3)(\mathrm{p} 36.3 ; \mathrm{q} 25)$ involving a fusion transcript where exon 4 of WWTR1 is fused in frame with either exon 8 or exon 9 of CAMTA1 [4]. An alternative fusion transcript of YAP1-TFE3 or novel WWTR1CAMTA1 fusion variants have also been observed and may bear significance on clinical behavior and variant morphology $[5,6]$. CAMTA1 is a transcription factor and putative tumor suppressor [7]. One of this WC fusion protein subsequently leads to oncogenic transformation and resistance to anoikis (anchorage-dependent cell death) [7]. Molecular genetic methods, for example RT-PCR or fluorescent in situ hybridization (FISH), are the gold standard for detection of tumor-specific genetic alterations. Recently, nuclear CAMTA1 expression has been found to be a useful immunohistochemical marker and can distinguish EHE from its histologic mimics $[8,9]$. Up to one-half of EHE cases show multifocal presentation even in different locations [10]. Utilizing molecular analysis to understand disease progression, multifocal EHEs probably represent metastatic implants of the primary tumor rather than synchronous multiple tumor clones [10]. Consistent with the above concept, the cecal tumor in our case was considered to be secondary to the hepatic EHE, although the primary tumor tissue was unavailable for molecular analysis.

There is no standard treatment for hepatic EHE because of its rarity and variable clinical outcome. The decision on a treatment strategy should be individualized for each patient. Surgical resection is the treatment of choice for localized liver involvement, and liver transplantation is reasonable when the liver parenchyma is diffusely involved. Adjuvant chemotherapy is considered when extrahepatic involvement occurs [11]. An optional choice is trans-arterial chemoembolization with chemotherapy and/or radiotherapy, although EHE may be poorly responsive to both therapies. Palliative resection is not advocated because the tumor behaves aggressively after liver resection [11]. Recently, a novel alternative therapy targeting angiogenesis, for e.g. thalidomide or metronomic cyclophosphamide, has been found to be promising [12-14]. The 5-year survival rates for hepatic EHE patients with liver resection, chemotherapy or radiotherapy, and no treatment are $75 \%, 30 \%$ and $5 \%$ respectively [11]. Liver transplantation for multicentric liver tumors yields a 5 -year survival rate of $55 \%$ [11]. The overall 5-year survival rate of EHE is $41 \%$. Our case demonstrated a natural disease course of hepatic EHE with a survival of more than 3.3 years.

\section{Acknowledgement}

We are grateful to Dr. Michael W Hughes (Assistant Professor, Institute of Clinical Medicine, College of Medicine, National Cheng Kung University) for critical reviewing the manuscript.

\section{References}

1. Alvarez Sanchez JA, Fernandez Lobato R, Coba Ceballos J, Fradejas Lopez JM, Marin Lucas J, et al. (1995) Epithelioid hemangioendothelioma localized in the small intestine. Gastroenterol Hepatol 18: 464-467.

2. Arena V, Pennacchia I, Carbone A, Vecchio FM (2011) Epithelioid hemangioendothelioma of the bone and intestinal infarction: An unusual association. Colorectal Dis 13: 69-70.

3. Mistry AM, Gorden DL, Busler JF, Coogan AC, Kelly BS (2012) Diagnostic and therapeutic challenges in hepatic epithelioid hemangioendothelioma. J Gastrointest Cancer 43: 521-525.

4. Errani C, Zhang L, Sung YS, Hajdu M, Singer S, et al. (2011) A novel WWTR1-CAMTA1 gene fusion is a consistent abnormality in epithelioid hemangioendothelioma of different anatomic sites. Genes Chromosomes Cancer 50: 644-653.

5. Antonescu CR, Le Loarer F, Mosquera JM, Sboner A, Zhang L, et al. (2013) Novel YAP1-TFE3 fusion defines a distinct subset of epithelioid hemangioendothelioma. Genes Chromosomes Cancer 52: 775-784.

6. Patel NR, Salim AA, Sayeed H, Sarabia SF, Hollingsworth F, et al. (2015) Molecular characterization of epithelioid haemangioendotheliomas identifies novel WWTR1-CAMTA1 fusion variants. Histopathology 67: 699-708.

7. Tanas MR, Ma S, Jadaan FO, Ng CK, Weigelt B, et al. (2016) Mechanism of action of a WWTR1(TAZ)-CAMTA1 fusion oncoprotein. Oncogene 35: 929-38.

8. Doyle LA, Fletcher CD, Hornick JL (2016) Nuclear expression of CAMTA1 distinguishes epithelioid hemangioendothelioma from histologic mimics. Am J Surg Pathol 40: 94-102.

9. Shibuya R, Matsuyama A, Shiba E, Harada H, Yabuki K, et al. (2015) CAMTA1 is a useful immunohistochemical marker for diagnosing epithelioid haemangioendothelioma. Histopathology 67: 827-35.

10. Errani C, Sung YS, Zhang L, Healey JH, Antonescu CR (2012) Monoclonality of multifocal epithelioid hemangioendothelioma of the liver by analysis of WWTR1-CAMTA1 breakpoints. Cancer Genet 205: 12-17.

11. Mehrabi A, Kashfi A, Fonouni H, Schemmer P, Schmied BM, et al. (2006) Primary malignant hepatic epithelioid hemangioendothelioma: A comprehensive review of the literature with emphasis on the surgical therapy. Cancer 107: 2108-2121.

12. Lakkis Z, Kim S, Delabrousse E, Jary M, Nguyen T, et al. (2013) Metronomic cyclophosphamide: an alternative treatment for hepatic epithelioid hemangioendothelioma. J Hepatol 58: 1254-1257.

13. Mascarenhas RC, Sanghvi AN, Friedlander L, Geyer SJ, Beasley HS, et al. (2004) Thalidomide inhibits the growth and progression of hepatic epithelioid hemangioendothelioma. Oncology 67: 471-475.

14. Soape MP, Verma R, Payne JD, Wachtel M, Hardwicke F, et al. (2015) Treatment of Hepatic Epithelioid Hemangioendothelioma: Finding Uses for Thalidomide in a New Era of Medicine. Case Rep Gastrointest Med. 